Valquiria Maria de Paula Cunha ${ }^{1}$

Roseane Lopes da Silva Grecco ${ }^{2}$

Marina CarValho Pascholn ${ }^{3}$ SUELI RIUL DA SILVA ${ }^{4}$

MARIÂNGELA TORREGLOSA RUIZ ${ }^{5}$ MARLy APARECIDA SPADOTTO BALARIN ${ }^{6}$

\section{Artigo original}

Palavras-chave

Fatores de crescimento do endotélio vascular

Gravidez

Hipertensão

Polimorfismo genético

Pré-eclâmpsia

Keywords

Vascular endothelial growth factors

Pregnancy

Hypertension

Polymorphism, genetic

Pre-eclampsia

\title{
Polimorfismos genéticos do fator de crescimento do endotélio vascular na pré-eclâmpsia
}

\author{
Genetic polymorphisms of vascular endothelial growth factor in \\ pre-eclampsia
}

\section{Resumo}

OBJETIVO: Identificar polimorfismos genéticos do fator de crescimento do endotélio vascular (VEGF), posições +936C/T e-2578C/A, em mulheres com pré-eclâmpsia.MÉTODOS:Trata-se de um estudo transversal, constituído por 80 mulheres distribuídas em dois grupos: pré-eclâmpsia e grupo controle. A caracterização da amostra foi realizada mediante entrevista pré-estruturadae complementada por dados transcritos dos prontuários. Para identificação dos polimorfismos foi realizada extração de DNA, amplificação das sequências pela Reação em Cadeia da Polimerase (PCR) com primers específicos e análise por Polimorfismos de Comprimentos de Fragmentos de Restrição (RFLP). A análise estatística dos resultados foi realizada de forma descritiva e pelo teste do $\chi^{2}$. $\bigcirc$ modelo de regressão logística múltipla foi utilizado para determinar o efeito dos polimorfismos na pré-eclampsia. RESULTADOS: Evidenciou-se uma maior frequência do alelo T do polimorfismo VEGF +936C/T nas pacientes com pré-eclâmpsia, embora com diferença não significativa.A presença do alelo A do VEGF -2578C/A foi maior no grupo controle, com diferença significativa. CONCLUSÕES:Não foi observada associação significativa do polimorfismo VEGF $+936 \mathrm{C} / T$ com a pré-eclâmpsia. Para o polimorfismo VEGF -2578C/A observa-se diferença significativa entre os grupos, sendo o alelo A mais frequente no controle, sugerindo a possibilidade da portadora do alelo A apresentar menor suscetibilidade para o desenvolvimento de pré-eclâmpsia.

\section{Abstract}

PURPOSE:To identify genetic polymorphisms of endothelial growth factor (VEGF), positions $+936 \mathrm{C} / \mathrm{T}$ and $-2578 \mathrm{C} / \mathrm{A}$, in women with pre-eclampsia. METHODS:This was a cross-sectional study conducted on 80 women divided into two groups: pre-eclampsia and control. The sample was characterized using a pre-structured interview and data transcribed from the medical records. DNA extraction, amplification of sequences by the Polymerase Chain Reaction (PCR) with specific primers and polymorphism analysis of Restriction Fragment Length Polymorphism (RFLP) were performed to identify polymorphisms. The statistical analysis was performedin a descriptive manner and using the $\chi^{2}$ test. The multiple logistic regression model was used to determine the effect of polymorphisms on pre-eclampsia. RESULTS:Ahigher frequency of the T allele of theVEGF $+936 \mathrm{C} / \mathrm{T}$ polymorphism was observedin patients with pre-eclampsia, but with no significant difference. The presence of allele A of the VEGF -2578C/A was significantly higher in the control group. CONCLUSIONS:No significant association was observed between VEGF +936C/Tpolymorphism andpre-eclampsia. For the VEGF -2578C/A polymorphism a significant differencewas observed between thecontrol and pre-eclampsia group, with allele A being the most frequent in the control, suggesting the possibility that carriers of allele A have lower susceptibility to the development of pre-eclampsia.

Correspondência:

Marly Aparecida Spadotto Balarin Disciplina de Genética - Universidade Federal do Triñngulo Mineiro Praça Manoel Terra, 330 (EP: $38015-050$ - Uberaba (MG), Brasil

Recebido $25 / 11 / 2010$
Disciplina de Genética da Universidade Federal do Triângulo Mineiro - UFTM - Uberaba (MG), Brasil.

'Mestre em Atenção à Saúde pela Universidade Federal do Triângulo Mineiro - UFTM - Uberaba (MG), Brasil.

2Professora Assistente II da disciplina de Genética daUniversidade Federal do Triângulo Mineiro - UFTM - Uberaba (MG), Brasil. 3Professora Adjuntado Departamento Materno-Infantil da Universidade Federal do Triângulo Mineiro - UFTM - Uberaba (MG), Brasil. 4Professora Associada I da Universidade Federal do Triângulo Mineiro - UFTM - Uberaba (MG), Brasil.

5Professora Adjuntal da disciplina de Biologia Molecular e Genética da Universidade Federal do Triângulo Mineiro - UFTM - Uberaba (MG), Brasil.

'Professora Associada III da disciplina de Genética da Universidade Federal do Triângulo Mineiro - UFTM - Uberaba (MG), Brasil. 


\section{Introdução}

A pré-eclâmpsia (PE) é uma síndrome multissistêmica específica da gravidez, definida clinicamente como pressão arterial aumentada $(>140 / 90 \mathrm{mmHg})$ e proteinúria que excede $300 \mathrm{mg} /$ dia após a vigésima semana de gestação, podendo cursar também com edema ${ }^{1,2}$. Afeta de 5 a $8 \%$ das grávidas, sendo a principal causa de morbidade e mortalidade materno-fetal ${ }^{2-5}$.

Os fatores de risco incluem: primíparas, multíparas com novo parceiro, $\mathrm{PE}$ em gestações anteriores e idade materna (extremos do período reprodutivo). Algumas condições obstétricas que aumentam a massa placentária como gestação múltipla e mola hidatiforme também contribuem para o maior risco de $\mathrm{PE}^{6-9}$. O diagnóstico precoce e a intervenção são de importância vital em reduzir a mortalidade materna e perinatal ${ }^{10}$.

Apesar de existirem muitas pesquisas nessa área, a patogênese da PE é pouco compreendida ${ }^{1,3}$. Possíveis etiologias têm sido propostas, incluindo causas placentárias, imunológicas, genéticas e ambientais. Alguns estudos sugerem que a PE está relacionada ao desequilíbrio de fatores angiogênicos circulantes, resultando em disfunção endotelial ${ }^{1,11,12}$.

O progresso na prevenção e no tratamento desta condição requer avanços na compreensão da fisiopatologia da doença em nível molecular ${ }^{10}$. A descoberta de novos biomarcadores para detectar essa síndrome, antes das manifestações clínicas é uma proposta promissora, sendo que a genética pode contribuir com essas novas opções ${ }^{3,13,14}$. Entre os genes candidatos estão incluídos aqueles que codificam fatores da coagulação, estresse oxidativo e substâncias vasoativas, bem comoos envolvidos na função placentária e citocinas inflamatórias ${ }^{10,15}$.

O fator de crescimento do endotélio vascular (VEGF) é um importante fator angiogênico, produzido por macrófagos, linfócitos T, células tumorais e pelo citotrofoblasto, e representa papel fundamental na regulação da implantação da placenta através dos seus efeitos na remodelação vascular e na sobrevivência e invasão do citotrofoblasto ${ }^{16}$.

O gene que codifica VEGF é altamente polimórfico, está localizado no cromossomo $6 \mathrm{p} 21.3$ e contém $14 \mathrm{~kb}$ na região codificante com oito éxons e sete íntrons ${ }^{17}$. Os polimorfismos VEGF podem ser úteis como indicadores de suscetibilidade para $\mathrm{PE}^{18}$. Baseado na predisposição genética, esta relação pode ser fortemente mostrada pela associação entre polimorfismos genéticos e aumento de risco para o desenvolvimento de $\mathrm{PE}^{19}$. Alguns desses, tais como VEGF + 936C/T e VEGF -2578C/A podem ter um impacto na produção do VEGF, sendo que sua diminuição predispõe risco aumentado para $\mathrm{PE}^{16,18,20}$.

Considerando-se a PE como um dos principais problemas da prática obstétrica e a importância do VEGF para a gravidez, este estudo teve o objetivo de identificar polimorfismos do gene VEGF nas posições -2578C/A e +936C/T em mulheres com PE.

\section{Métodos}

Trata-se de um estudo transversal e quantitativo,no qual foram incluídas por 80 mulheres distribuídas em dois grupos: pré-eclâmpsia e grupo controle. Todas foram atendidas no Hospital de Clínicas da Universidade Federal do Triângulo Mineiro (HC-UFTM), no período de agosto de 2008 a dezembro de 2009. No grupo caso, foram incluídas 52 mulheres, com idade igual ou superior a 18 anos, que apresentaram PE durante a gestação, verificadapelo exame de proteinúria e classificadas de acordo com o consenso do National High Blood Pressure Education Program (NHBPEP, 2000). O grupo controle foi composto por 28 mulheres com idade igual ou superior a 18 anos que apresentaram duas ou mais gestações sem intercorrências, com filhos normais. Foram excluídas mulheres com idade inferior a 18 anos e no grupo controle, aquelas com síndromes hipertensivas e outras complicações. A caracterização da amostra foi realizada mediante entrevista pré-estruturada com os sujeitos da pesquisa e complementada por dados transcritos dos prontuários. As variáveis analisadas foram: idade, etnia, número médio de gestações, tabagismo, etilismo, presença de doença crônica e pré-natal.

A extração do DNA foi realizada pelo método de fenol/clorofórmio. Para amplificação das sequências, as amostras de DNA genômico foram submetidas à Reação em Cadeia da Polimerase (PCR), utilizando-se pares de primers: VEGF +936C/T - sense: 5'AAGGAAGAGGAGACTCTGCGCAGAGC - 3' e antisense: 5'-AAATGTATGTATGTGGGTGGGTGTGTCTACAGG-3'; VEGF -2578C/A - sense: 5'GGGCCTTAGGACACCATACC-3' e antisense: 5'TGCCCCAGGGAACAAAGT-3'. A amplificação do produto de PCR das amostras foi visualizada por meio de eletroforese em gel de agarose $3 \%$ e coloração pelo GelRed (Biotium ${ }^{\circledR}$ ).

Os produtos da PCR foram submetidos à digestão por enzimas de restrição específicas para cada polimorfismo em estudo: VEGF +936C/T pela enzima NlaIII $\left(\right.$ BioLabs $\left.^{\circledR}\right)$ e VEGF -2578C/A pela enzima $B g l$ II $\left(\right.$ BioLabs $\left.^{\circledR}\right)$, por 3 h a $37^{\circ} \mathrm{C}$. Em relação ao polimorfismo $+936 \mathrm{C} / \mathrm{T}$, para o alelo $\mathrm{C}$ formou-se um fragmento de $208 \mathrm{pb}$ e para o T, 122 e 86 pb. Já para o -2578C/A, o alelo C, 267pb e o A 208 e $60 \mathrm{pb}$.

O produto de digestão foi aplicado em gel de poliacrilamida $10 \%$ e os fragmentos descritos foram separados em eletroforese, corados pela prata e as imagens foram obtidas pelo Sistema de Captura de Imagens (L-PIX ${ }^{\circledR}$ ). A análise foi feita pelo tamanho dos fragmentos de restrição. 
Para a análise estatística, os dados foram compilados no software Excel $^{\circledR}$ 4.0, utilizando-se sua planilha eletrônica para armazenamento. A análise estatística dos resultados foi realizada de forma descritiva e inferencial. Para variáveis quantitativas, utilizaram-se medidas de posição ou centralidade (média) e medidas de dispersão e variabilidade (desvio padrão). As variáveis categóricas foram analisadas por meio do software StatView ${ }^{\circledR}$ empregando-se frequências relativas, bem como, análise de associação em tabelas de contingências $\chi^{2}$. O equilíbrio de Hardy-Weinberg (HWE) foi realizado pelo teste do $\chi^{2}$. O modelo de regressão logística múltipla foi utilizado para determinar o efeito dos polimorfismos na PE, tendo como referência a ausência do polimorfismo.Os valores foram considerados estatisticamente significativos quando $\mathrm{p} \leq 0,05$.

O projeto foi aprovado pelo Comitê de Ética em Pesquisa da Universidade Federal do Triângulo Mineiro -Protocolo CEP no 1115-08 em 18 de abril de 2008, e todas as mulheres assinaram o termo de consentimento livre e esclarecido.

\section{Resultados}

O grupo caso foi composto por 52 pacientes $(65 \%$ da amostra) portadoras de PE com média de idade de $26,9 \pm 6,4$ anos. No que se refere à etnia, $7(13,5 \%)$ eram negras e $45(86,5 \%)$ não negras. A média de gestações entre as mulheres do grupo foi de $1,98 \pm 1,20$, sendo que $23(44,2 \%)$ eram primíparas, $46(88,5 \%)$ não tabagistas e $4(7,7 \%)$ apresentavam alguma doença crônica (hipertensão arterial crônica, glaucoma e hipotireoidismo). Todas as mulheres $(100 \%)$ fizeram pré-natal com média de sete consultas. Não houve relato de etilismo, uso de drogas ou gestação múltipla. Na amostra, 12 (23\%) tinham história de aborto.

Todas as pacientes apresentaram proteinúria $>300 \mathrm{mg}$ em urina de $24 \mathrm{~h}$ ou pelo menos ++ no teste de fita (dipstick). A média da pressão arterial sistólica foi de $157 \pm 23 \mathrm{mmHg}$ e da diastólica $105 \pm 16 \mathrm{mmHg}$. Dentre algumas complicações maternas ocorreram, em sete mulheres $(13,5 \%)$ a síndrome HELLP, em três $(5,8 \%)$ iminência de eclâmpsia e duas $(3,8 \%)$ evoluíram para eclâmpsia pré-parto.

A maioria das gestações do grupo com PE encerrou-se por partos cesarianos e apenas seis $(11,5 \%)$ por partos vaginais. Ao que se refere à idade gestacional no momento do parto, $19(36,5 \%)$ estavam com menos de 34 semanas, $19(36,5 \%)$ entre 34 a 37 semanas e $13(25 \%)$ mais que 37 semanas.

Neste grupo, 40 (77\%) mulheres referiram história familiar de hipertensão e/ou hipertensão na gestação e as demais 12 (23\%) desconhecem a informação ou não houve casos na família. Os relatos de hipertensão específica da gestação na família foram de $29(55,8 \%)$.
O grupo controle foi constituído por 28 mulheres (35\% da amostra) que apresentaram duas ou mais gestações sem intercorrências, com filhos saudáveis. A média de idade foi de 25,1 $1 \pm 5,31$ anos e 28,6\% eram negras.

A Figura 1 mostra o gel de poliacrilamida com os genótipos T/T, C/T e CC do polimorfismo do gene VEGF posição $+936 \mathrm{C} / \mathrm{T}$.As frequências dos genótipos $\mathrm{C} / \mathrm{C}, \mathrm{C} / \mathrm{T}$ e T/T do polimorfismo do gene VEGF +936C/T no grupo PE foram 65,0, 31,0 e 4,0\%, respectivamente. No grupo controle,foram 78,6\% (C/C), 17,8\% (C/T) e 3,6\% (T/T). As diferenças não foram estatisticamente significativas $\left(\chi^{2}=1,6\right.$; $\mathrm{p}=0,4)$ (Tabela 1).Considerando-se a presença ou não do alelo $T$, não foi observada diferença significativa $\left(\chi^{2}=1,5\right.$; $\mathrm{p}=0,2)$, apesar do mesmo apresentar maior frequência nas portadoras de PE (35\%). As frequências genotípicas encontram-se em equilíbrio de Hardy-Weinberg entre as pacientes $\left(\chi^{2}=0,005 ; \mathrm{p}=0,95\right)$ e entre os indivíduos controles $\left(\chi^{2}=0,95 ; \mathrm{p}=0,33\right)$.

$\mathrm{Na}$ Figura 2, observam-se os genótipos C/C, C/A e A/A do polimorfismo do gene VEGF posição $-2578 \mathrm{C} / \mathrm{A}$. As frequências desses genótipos foram 58,0, 31,0 e $11,0 \%$ no grupo PE. Já no grupo controle, foram $33,0 \%$ (C/C),60,0\% (C/A) e 7,0\% (A/A). As diferenças foram estatisticamente significativas $\left(\chi^{2}=6 ; p=0,049\right)$ (Tabela 2).Considerando-se a presença ou não do alelo A, foi observada diferença estatisticamente significativa $\left(\chi^{2}=4,2 ; p=0,04\right)$, sendo mais frequente no grupo controle $(67 \%)$.As frequências genotípicas encontraram-se em equilíbrio de Hardy-Weinberg entre as pacientes

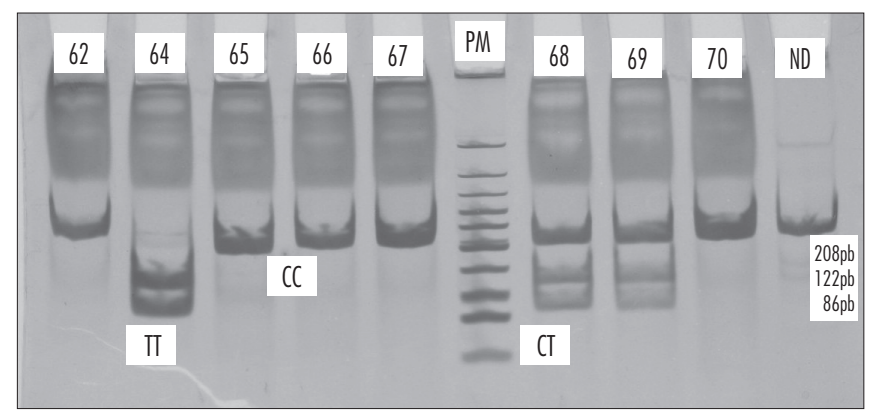

Figura 1. Gel de poliacrilamida 10\% com os produtos de PCR (208pb), do polimorfismo VEGF +936C/T, digeridos pela enzima de restrição Nlalll, mostrando os genótipos $\mathrm{T} / \mathrm{T}, \mathrm{C} / \mathrm{T}$ e CC. Peso Molecular $(\mathrm{PM})=25 \mathrm{pb}$; produto não digerido (ND).

Tabela 1. Frequência dos genótipos do polimorfismo VEGF +936C/T,nos grupospréeclâmpsiae controle

\begin{tabular}{|c|c|c|c|c|c|c|c|c|}
\hline \multirow{3}{*}{ Condição } & \multicolumn{6}{|c|}{ Genótipos } & \multirow{2}{*}{\multicolumn{2}{|c|}{ Total }} \\
\hline & \multicolumn{2}{|c|}{$C / C$} & \multicolumn{2}{|c|}{$\mathrm{C} / \mathrm{T}$} & \multicolumn{2}{|c|}{$\mathrm{T} / \mathrm{T}$} & & \\
\hline & $n$ & $\%$ & $n$ & $\%$ & $n$ & $\%$ & $n$ & $\%$ \\
\hline PE & 34 & 65,0 & 16 & 31,0 & 2 & 4,0 & 52 & 100 \\
\hline C & 22 & 78,6 & 5 & 17,8 & 1 & 3,6 & 28 & 100 \\
\hline Total & 56 & 70 & 21 & 26,3 & 3 & 3,7 & 80 & 100 \\
\hline
\end{tabular}

PE - pré-eclâmpsia; $C$ - controle; VEGF - fator de crescimento do endotélio vascular; $\chi^{2}=1,6 ; p=0,4$ 
$\left(\chi^{2}=0,15 ; \mathrm{p}=0,70\right)$ e entre os indivíduos controles $\left(\chi^{2}=1,98 ; \mathrm{p}=0,70\right)$.

Analisando-sea combinação genotípica dos polimorfismos do gene VEGF + 936C/A e VEGF -2578C/A, observa-se uma frequência maior da combinação CCCA, no total da amostra (32\%). Considerando-se os grupos separadamente, a combinação mais frequente na PE foi CCCC $(34,6 \%)$ e no grupo controle foi CCCA $(50 \%)$ (Tabela 3).

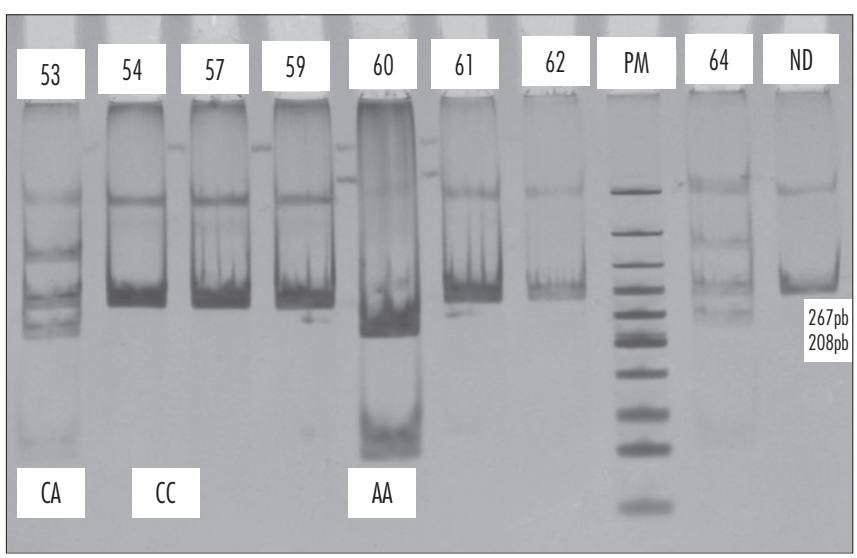

Figura 2. Gel de poliacrilamida 10\% com os produtos de PCR (267pb), do polimorfismo VEGF -2578 C/A, digeridos pela enzima de restrição Bglll, mostrando os genótipos A/A, C/A e C/C. Peso Molecular (PM)=25pb; produto não digerido (ND).

Tabela 2. Frequência dos genótipos do polimorfismo VEGF-2578 C/A, nos grupospéeclâmpsiae controle

\begin{tabular}{|c|c|c|c|c|c|c|c|c|}
\hline \multirow{3}{*}{ Condição } & \multicolumn{6}{|c|}{ Genótipos } & \multirow{2}{*}{\multicolumn{2}{|c|}{ Total }} \\
\hline & \multicolumn{2}{|c|}{$C / C$} & \multicolumn{2}{|c|}{ C/A } & \multicolumn{2}{|c|}{$A / A$} & & \\
\hline & $n$ & $\%$ & $\mathrm{n}$ & $\%$ & $\mathrm{n}$ & $\%$ & $n$ & $\%$ \\
\hline $\mathrm{PE}$ & 30 & 58 & 16 & 31 & 6 & 11 & 52 & 100 \\
\hline C & 9 & 33 & 16 & 60 & 2 & 7 & 27 & 100 \\
\hline Total & 39 & 49 & 32 & 41 & 8 & 10 & 79 & 100 \\
\hline
\end{tabular}

PE - pré-eclâmpsia; C - controle;VEGF - fator de crescimento do endotélio vascular; $\chi^{2}=6 ; p=0,049$.

Tabela 3. Frequência da combinacão genotípica dos polimorfismos VEGF $+936 \mathrm{C} / \mathrm{T}$ e $-2578 \mathrm{C} / \mathrm{A}$ entre individuos dos grupospré-eclâmpsiae controle

\begin{tabular}{|c|c|c|c|c|c|c|}
\hline \multirow{2}{*}{$\begin{array}{c}\text { Combinação } \\
\text { genotípica } \\
\text { VEGF } \\
+936 /-2578\end{array}$} & \multicolumn{2}{|c|}{$\mathrm{PE}$} & \multicolumn{2}{|c|}{ C } & \multicolumn{2}{|c|}{ Total } \\
\hline & $n$ & $\%$ & n & $\%$ & n & $\%$ \\
\hline CCAA & 4 & 7,7 & 2 & 7,8 & 6 & 7,7 \\
\hline CCCA & 12 & 23,1 & 13 & 50 & 25 & 32 \\
\hline cccc & 18 & 34,6 & 6 & 23,1 & 24 & 31 \\
\hline СТАA & 2 & 3,8 & 0 & 0 & 2 & 2,5 \\
\hline СТCA & 3 & 6 & 1 & 3,8 & 4 & 5 \\
\hline CTCC & 11 & 21 & 3 & 11,5 & 14 & 18 \\
\hline TTAA & 0 & 0 & 0 & 0 & 0 & 0 \\
\hline TTCA & 1 & 1,9 & 1 & 3,8 & 2 & 2,5 \\
\hline TTCC & 1 & 1,9 & 0 & 0 & 1 & 1,3 \\
\hline Total & 52 & 100 & 26 & 100 & 78 & 100 \\
\hline
\end{tabular}

PE - pré-eclâmpsia; C - controle; VEGF - fator de crescimento do endotélio vascular.
$\mathrm{Na}$ análise de regressão logística múltipla, foram analisados os polimorfismos $+936 \mathrm{C} / \mathrm{T}$ e $-2578 \mathrm{C} / \mathrm{A}$ do gene VEGFem pacientes com PE e indivíduos controles e não foram encontradas diferenças entre os grupos para o polimorfismo $+936 \mathrm{C} / \mathrm{T}(\mathrm{OR}=1,95$; IC95\% $=0,91-6,25$; $\mathrm{p}=0,26)$ e para o polimorfismo $-2578 \mathrm{C} / \mathrm{A}(\mathrm{OR}=0,41$; IC95\% $=0,15-1,10 ; \mathrm{p}=0,08)$.

\section{Discussão}

OVEGF é um importante fator angiogênico e representa papel fundamental como regulador na proliferação celular endotelial e permeabilidade vascular. O gene que codifica o VEGF é altamente polimórfico e seus polimorfismos funcionais podem ser úteis como indicadores de suscetibilidade para a $\mathrm{PE}^{18}$.

A progressão da PE pode ser modificada na presença dos polimorfismos VEGF -2578C/A e +936C/T que predispõem a baixa capacidade de produção de $\mathrm{VEGF}^{16,18}$. No presente estudo,não foi observada diferença significativa para os genótipos (C/C, C/T e T/T) do gene VEGF +936C/T e para a presença do alelo T,provavelmente devido ao número reduzido de mulheres no grupo controle.No estudo de Shim et al. ${ }^{18}$, esse polimorfismo foi analisado em 110 pacientes com PE e 209 controles e se demonstrou aumento de susceptibilidade e fator de risco independente para o desenvolvimento de PE em portadoras do alelo T. Entretanto, outras autoras ${ }^{21}$ mostraram resultados contraditórios, sem encontrar diferença significativa, semelhante aos achados do presente estudo.

Em relação aos três genótipos $(\mathrm{C} / \mathrm{C}, \mathrm{C} / \mathrm{A}$ e $\mathrm{A} / \mathrm{A})$ do VEGF - 2578C/A, foram encontradasdiferenças estatisticamente significativas $\left(\chi^{2}=6 ; p=0,049\right)$. Sendo que o genótipo $\mathrm{C} / \mathrm{C}$ apresentou maior frequência na amostra total $(49 \%)$. Analisando-se os grupos separadamente encontraram-se frequências diferentes no que se refere ao tipo de genótipo, sendo que na $\mathrm{PE}$ o predominante foi C/C (58\%) e no controle o genótipo C/A (60\%). Em relação à presença do alelo $\mathrm{A}$, notou-se uma maior frequência no grupo controle $(67 \%)$, com diferença significativa.

Dados semelhantes foram encontrados em um estudo realizado na Hungria, onde se observou um percentual maior dos genótipos $\mathrm{C} / \mathrm{C}$ e $\mathrm{C} / \mathrm{A}$ nas pacientes com $\mathrm{PE}$ e no grupo controle, respectivamente. No entanto, o genótipo mais comum foi $\mathrm{C} / \mathrm{A}$ quando considerada a amostra total (grupo controle e PE) ${ }^{16}$. Considerando-se que o VEGF tem um papel importante na integridade da barreira de filtração glomerular, assim como a inibição de sua atividade pode levar a proteinúria, pode-se sugerir que, em portadoras do alelo $\mathrm{A}$, a proteinúria esta associada à baixa produção de $\operatorname{VEGF}^{20,22,23}$, o que é contraditório com os achados acima descritos. 
Apesar de o presente trabalho mostrar diferença significativa entre a frequência genotípica dos grupos, os genótipos observados diferem dos encontrados por outros autores. Em mulheres gregas, verificou-se uma frequência maior do genótipo C/A nos grupos controle e de PE, porém, sem diferença significativa ${ }^{21}$. No Brasil, em estudo constituído por três grupos: hipertensão gestacional, OE e controle, observou-se uma incidência maior do genótipo C/A na amostra total e nos grupos separadamente. Entretanto, essa diferença foi significativa somente quando foram excluídas as mulheres negras das análises ${ }^{24}$. Isto deve-se provavelmente pelo fato de que diferenças entre etnias podem influenciar na distribuição dos polimorfismos de VEGF.

Outros estudos revelam resultados contraditórios. Foi verificado que casos precoces de PE estavam mais presentes nas portadoras do alelo VEGF -2578A, sugerindo que a $\mathrm{PE}$ pode ser acelerada na presença deste polimorfismo ${ }^{16}$. Investigando-se a presença do alelo A em relação aos grupos controle, hipertensão gestacional e PE, encontrou-sefrequência maior desse alelo nas pacientes com hipertensão gestacional, no entanto, a diferença não foi significativa ${ }^{24}$.

Nas bases de dados pesquisadas (MEDLINE, LILACS e SCIELO) não foram encontrados artigos a respeito da combinação genotípica dos polimorfismos VEGF, posições +936C/T e -2578C/A, associados com PE. Entretanto, deve-se considerar que o gene VEGFestálocalizado em uma região do cromossomo 6 altamente polimórfica, e que a combinação deste comoutros genes, possivelmente associados àPE, deve ser analisada; o que justifica a análise de combinação genotípica.

Resultados conflitantes podem ser justificados por possíveis interferências, tais como: diferentes critérios diagnósticos da PE, número da amostra, desequilíbrio de ligação de genes, diferenças étnicas e heterogeneidade da população estudada ${ }^{18,23,25}$. Portanto, a composição de uma amostra maior, mais homogênea e a utilização deoutros polimorfismos genéticos, contribuirão para uma melhor identificação dos fatores genéticos envolvidos na PE.Embora, no presente estudo, não tenha sido encontrada associação significativa entre o polimorfismo VEGF na posição $+936 \mathrm{C} /$ Tcom a PE, o estudo dessas e outras variações genéticas permanece como um importante campo de pesquisa na busca da etiologia dessa síndrome.

Em estudos de associação genética,é necessário considerar algumas falhas, como possíveis vieses na seleção dos participantes, desconhecimento da função do gene e a necessidade de grandes amostras. Mesmo assim, resultados negativos devem ser considerados, bem como a realização de metanálises para validação dos achados.

Além disso, a população brasileira é muito heterogênea, resultante da mistura de diferentes etnias oriundas de vários continentes, como Europa, África, Ásia e América. Foi observadadiferença na distribuição dos polimorfismos VEGF em mulheres brancas quando comparadas com negras, sugerindo que essas diferenças podem, em parte, explicar como essa variabilidade genética pode influenciar na suscetibilidade das doenças. Em relação ao polimorfismo VEGF -2578C/A, verificou-se que o alelo Aera mais frequente em mulheres brancas em relação às negras ${ }^{25}$.

Neste estudo, observou-se que a distribuição dos genótipos do polimorfismo VEGF na posição -2578C/A foi significativamente diferente entre mulheres com $\mathrm{PE}$ e as do grupo controle, sendo a presença do alelo A mais frequente no grupo controle, sugerindo a possibilidade da portadora do alelo A apresentarcerta proteção para o desenvolvimento de PE.

\section{Referências}

1. Shenoy V, Kanasaki K, Kalluri R. Pre-eclampsia: connecting angiogenic and metabolic pathways. Trends Endocrinol Metab. 2010;21(9):529-36.

2. Young BC, Levine RJ, Karumanchi SA. Pathogenesis of preeclampsia. Annu Rev Pathol. 2010;5:173-92.

3. Carty DM, Delles $C$, Dominiczak AF. Novel biomarkers for predicting preeclampsia. Trends Cardiovasc Med. 2008; 18(5):186-94.

4. Lam C, Lim KH, Karumanchi SA. Circulating angiogenic factors in the pathogenesis and prediction of preeclampsia. Hypertension. 2005; 46(5): 1077-85

5. Carty DM, Delles C, Dominiczak AF. Preeclampsia and future maternal health. J Hypertens. 2010;28(7):1349-55.

6. Koga K, Osuga Y, Tajima T, Hirota Y, Igarashi T, Fujii T, et al. Elevated serum soluble fms-like tyrosine kinase 1 (sFlt1) level in women with hydatiform mole. Fertil Steril. 2010;94(1):305-8.
7. Barton JR, Sibai BM. Prediction and prevention of recurrent preeclampsia. Obstet Gynecol. 2008; $112(2$ Pt 1):359-72.

8. Bdolah Y, Lam C, Rajakumar A, Shivalingappa V, Mutter W, Sachs BP, et al. Twin pregnancy and the risk of preeclampsia: bigger placenta or relative ischemia? Am J Obstet Gynecol. 2008; 198(4):428.e 1-6

9. Duckitt $H$, Harrington D. Risk factors for pre-eclampsia at antenatal booking: systematic review of controlled studies. BM. $2005 ; 330(7491): 565$.

10. Chappell S, Morgan L. Searching for genetic clues to the causes of pre-eclampsia. Clin Sci (Lond). 2006; $110(4): 443-58$.

11. Thadhani R, Mutter WP, Wolf M, Levine RJ, Taylor RN, Sukhatme $V P$, et al. First trimester placental growth factor and soluble fmslike tyrosine kinase 1 and risk for preeclampsia. J Clin Endocrinol Metab. 2004;89(2):770-5. 
12. Said J, Dekker G. Pre-eclampsia and thrombophilia. Best Pract Res Clin Obstet Gynaecol. 2003;17(3):441-58.

13. Hertig A, Liere P. New markers in preeclampsia. Clin Chim Acta. 2010;411(21-22):1591-5.

14. Gadonski G, Irigoyen MCC. Aspectos fisiológicos da hipertensão arterial na gravidez. Hipertensão. 2008;1 1(1):4-8.

15. Lachmeijer AM, Dekker GA, Pals G, Aarnoudse JG, ten Kate LP, Arngrimsson R. Searching for preeclampsia genes: the current position. Eur J Obstet Gynecol Reprod Biol. 2002;105(2):94-113.

16. Bányász I, Szabó S, Bokodi G, Vannay A, Vásárhelyi B, Szabó A, et al. Genetic polymorphisms of vascular endothelial growth factor in severe pre-eclampsia. Mol Hum Reprod. 2006; 12(4):233-6.

17. Mohammadi M, Ollier WE, Hutchinson IV. A functional association study of VEGF gene promoter polymorphisms with VEGF expression by stimulated pbm cells. Hum Immunol. 2003;64(10) Suppl $1: S 125$.

18. Shim JY, Jun JK, Jung BK, Kim SH, Won HS, Lee PR, et al. Vascular endothelial growth factor gene $+936 \mathrm{C} / \mathrm{T}$ polymorphism is associated with preeclampsia in Korean women. Am J Obstet Gynecol. 2007; 197(3):271.e1-4.

19. Cnattingius S, Reilly M, Pawitan Y, Lichtenstein P. Maternal and fetal genetic factors account for most of familial aggregation of preeclampsia: a population-based Swedish cohort study. Am J Med Genet A. 2004;130A(4):365-71.

20. Shahbazi M, Fryer AA, Pravica V, Brogan IJ, Ramsay HM, Hutchinson IV, et al. Vascular endothelial growth factor gene polymorphisms are associated with acute renal allograft rejection. J Am Soc Nephrol. 2002; 13(1):260-4.

21. Papazoglou D, Galazios G, Koukourakis MI, Panagopoulos I, Kontomanolis EN, Papatheodorou K, et al. Vascular endothelial growth factor gene polymorphisms and pre-eclampsia. Mol Hum Reprod.2004;10(5):321-4.

22. Eremina V, Quaggin SE. The role of VEGF-A in glomerular development and function. Curr Opin Nephrol Hypertens. 2004; 13(1):9-15.

23. Bdolah Y, Sukhatme VP, Karumanchi SA. Angiogenic imbalance in the pathophysiology of preeclampsia: newer insights. Semin Nephrol. 2004;24(6):548-56.

24. Sandrim VC, Palei AC, Cavalli RC, Araújo FM, Ramos ES, Duarte $G$, et al. Vascular endothelial growth factor genotypes and haplotypes are associated with pre-eclampsia but not with gestational hypertension. Mol Hum Reprod.2009; 15(2): 1 15-20.

25. Muniz JJ, Izidoro-Toledo TC, Metzger IF, Sandrim VC, Tanus-Santos JE. Interethnic differences in the distribution of clinically relevant vascular endothelial growth factor genetic polymorphisms. DNA Cell Biol. 2009;28(1 1):567-72. 